\title{
The influence of the form of condensation nucleus on the formation and propagation of acid precipitation near operating TPS
}

\author{
D.V. Gvozdyakov and V.E. Gubin
}

Tomsk Polytechnic University, 634050 Tomsk, Russia

\begin{abstract}
The results of numerical studies of condensation on the surface of the air component of atmospheric condensation nuclei of various shapes. Mathematical modeling performed at ambient temperatures typical of summer and winter seasons. It is found that the trajectory of motion of particles of various shapes differ significantly. Numerical studies were carried out at atmospheric parameters corresponding airspace adjacent to the areas of the TPP. Testing results obtained audited conservative difference scheme.
\end{abstract}

The Earth atmosphere currently represents the assortment of gaseous compounds of natural [1] (oxygen, nitrogen, etc.) and artificial (nitrogen oxides, sulfur anhydride, etc.) origin [1,2].

The geometry of atmospheric droplets are known to suffer numerous alterations during the movement [3]. The droplets having the diameter from $10^{-6}$ to $10^{-4}$ meters are generally spherical [4]. If the diameter is in the range from $10^{-4}$ to $10^{-3}$ meters, then the shape is ellipsoid. According to the results reported in [4], the geometry parameters of droplets affect the areal of their precipitation. This is caused by a dissimilar movement of aspherical and spherical droplets. Such difference is expressed both by quantitative characteristics (larger drag coefficient, $C_{D}$ ) and by the mechanism of the movement.

Previous studies [5] elucidate the formation mechanism of sediment acid precipitation in the atmosphere adjacent to a thermal power station.

The aim of the present study is to perform a numerical analysis of the formation of sediment acid precipitation in the atmosphere surrounding a thermal power station. The analysis is to consider the processes taking place on the surface of cylindrical and spherical condensation nuclei with a due regard of the main significant factors.

The analysis covers the problem, which solution region corresponds to the atmosphere around the source of air emission of products formed after the combustion of solid fuels containing $\mathrm{SO}_{3}$. The mechanism of formation of sediment droplets is similar to that reported in [6].

The solution of the problem assumes that each trajectory of the movement towards the Earth's surface corresponds to a single acid droplet. Moreover, its formation consumes the whole amount of $\mathrm{SO}_{3}$ available in the area surrounding the trajectory. The influence of aspheric form of the droplet was characterized by the asphericity coefficient $\Gamma$. The coefficient is determined as the relation of surface area of a real droplet to the surface area of a sphere with equal volume.

This is an Open Access article distributed under the terms of the Creative Commons Attribution License 4.0, which permits unrestricted use, distribution, and reproduction in any medium, provided the original work is properly cited. 


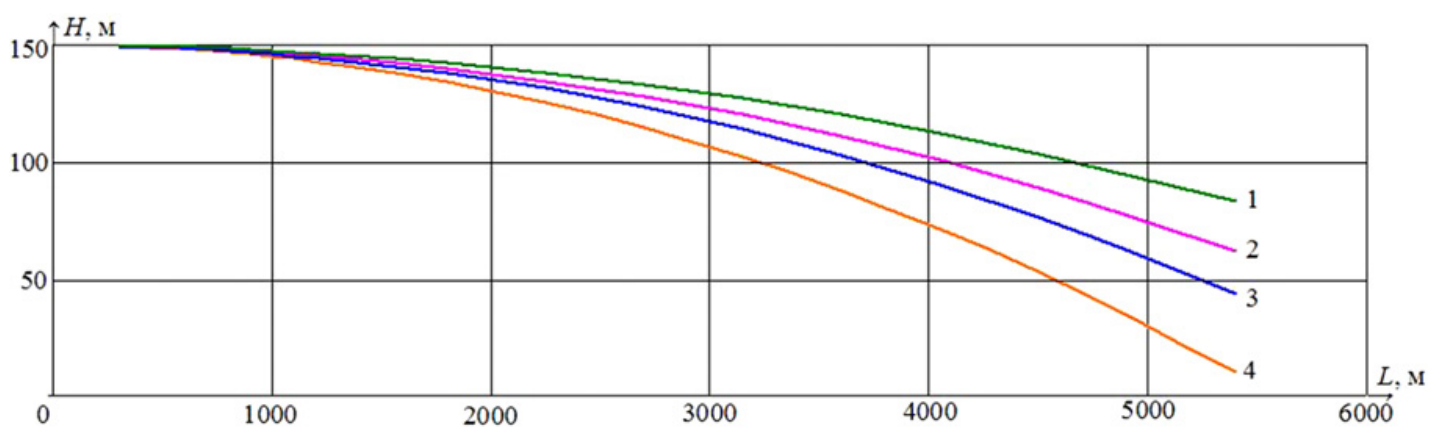

Figure 1. Droplet movement trajectories 1) and 2) are cylindrical droplets in winter and in summer, 3) and 4) are spherical droplets in winter and in summer.

The equations expressing heat conductivity and movement of a droplet (cylindrical and spherical) in the frame of the assumed physical model of the process under study are as follows.

$$
\left.\begin{array}{c}
\rho_{1} \cdot C_{P} \cdot \frac{\partial T_{1}}{\partial t}=\frac{\lambda}{r} \cdot \frac{\partial}{\partial r} \cdot\left(r \cdot \frac{\partial T_{1}}{\partial r}\right), \\
\rho_{1} \cdot C_{P} \cdot \frac{\partial T_{1}}{\partial t}=\lambda\left(\frac{\partial^{2} T_{1}}{\partial r^{2}}+\frac{2}{r} \cdot \frac{\partial T_{1}}{\partial r}\right), \\
\frac{d V_{X}}{d t}=\frac{3 \cdot \Gamma \cdot C_{D} \cdot \rho_{1} \cdot\left(V_{X}-U_{0}\right) \cdot\left(V_{X}-U_{0}\right)}{\mathbf{8} \cdot \pi \cdot \delta \cdot \rho_{2}} \\
\frac{d V_{Y}}{d t}=g+\frac{3 \cdot \Gamma \cdot C_{D} \cdot \rho_{1} \cdot\left(V_{Y}-V_{0}\right) \cdot\left(V_{Y}-V_{0}\right)}{\mathbf{8} \cdot \pi \cdot \delta \cdot \rho_{2}}
\end{array}\right\},
$$

where $\rho_{1,2}$ is density, $\mathrm{kg} / \mathrm{m}^{3} ; C_{P}$ is isobaric heat capacity, $\mathrm{J} /\left(\mu \mathrm{g} \cdot{ }^{\circ} \mathrm{C}\right) ; \lambda$ is the coefficient of heat conductivity, $\mathrm{W} /\left(\mathrm{m} \cdot{ }^{\circ} \mathrm{C}\right) ; T$ is temperature, $\mathrm{K} ; t$ is time, $\mathrm{s} ; V_{X}$ and $V_{Y}$ are the components of speed of a drop moving along $x$ and $y$ axes, respectively, $\mathrm{m} / \mathrm{s} ; C_{D}$ is a drag coefficient; $g$ is gravitational acceleration, $\mathrm{m} / \mathrm{s}^{2} ; r$ is a radius, $\mathrm{m} ; \delta$ is a specific geometrical size of a drop (Fig. 1), $\mathrm{m} ; U_{0}$ and $V_{0}$ are the components of speed of environment, $\mathrm{m} / \mathrm{s}$.

Initial and boundary conditions for Eqs. (1)-(2) have the following form.

$$
\left\{\begin{array}{l}
t=0: \quad T=T_{0}, \quad 0 \leq r \leq \delta \\
r=0: \quad-\lambda_{1} \cdot \frac{\partial T_{1}}{\partial r}+Q \cdot W_{K}=-\lambda_{2} \cdot \frac{\partial T_{2}}{\partial r}, \quad T_{1}=T_{2} ; \quad r=\frac{\delta}{2}: \quad \frac{\partial T_{1}}{\partial r}=0 .
\end{array}\right.
$$

where $\delta$ is the specific size of a drop, $\mathrm{m}$.

The speed of condensation was calculated using the Hertz-Knudsen equation [7].

We implemented the iteration algorithm that was developed for solving non-linear problems of heat conductivity under local thermal exposure $[8,9]$ by the method of finite difference. The reliability of the results of numerical modeling was verified by checking the conservativeness of a difference scheme by the method described in $[10,11]$. 

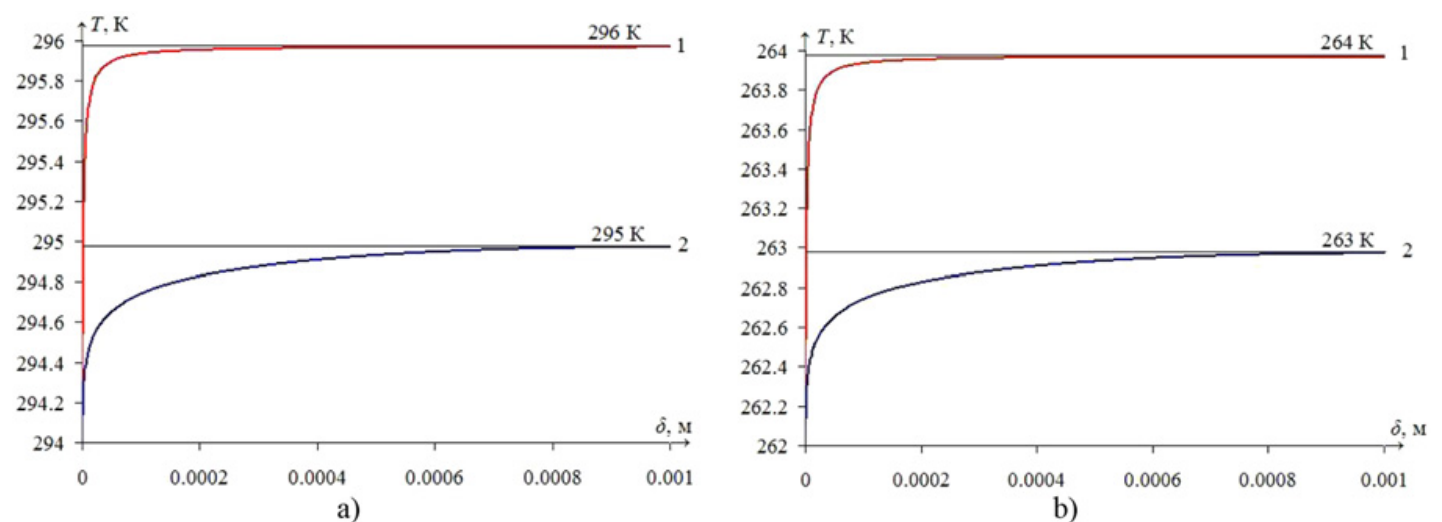

Figure 2. Temperature field of a droplet at the moment of $t=600$ seconds. a) in summer, b) in winter. 1) is spherical droplets, 2) is cylindrical droplets.

The mathematical modeling was carried out using the following values of the parameters that characterize the initial conditions of the process under consideration: $U_{0}=5 \mathrm{~m} / \mathrm{s} ; V_{0}=0.5 \mathrm{~m} / \mathrm{s}$. The temperature of a droplet at the initial moment was $294 \mathrm{~K}$ in summer and $262 \mathrm{~K}$ in winter.

The study of the influence of geometrical characteristics of formed droplets of acid was carried out for the time period of 6000 seconds. The droplets were developing on the surface of condensation nuclei having the size of $5 \cdot 10^{-6}$ meters.

Figure 1 depicts the trajectories of droplets in air in winter and in summer.

The results of numerical modeling show that the trajectories are significantly different for the particles having different form. In fact, over the considered time period in summer, the spherical particles reach the surface layers of the atmosphere (Fig. 1, curve 4). The cylindrical droplets over the time period of 6000 seconds are capable of propagating up to 5500 meters at the height from 50 to 100 meters.

The results of mathematical modeling (Fig. 2) allow drawing the following conclusions. Firstly, in the considered time period, the temperature of condensation surface (in winter and in summer) is comparable to that of atmospheric air. Secondly, over the considered period in summer, the droplets in surface layers are generally heated to the environmental temperature.

The work was carried out at the National Research Tomsk Polytechnic University within the Federal Target Program entitled "R\&D in priority areas of development of Russia's scientific and technological complex for 2014-2020" (unique ID: PNIER RFMEFI58114X0001).

\section{References}

[1] G.E. Zaikov, S.A. Maslov, V.L. Rubajlo, Acid rains and environment (1991)

[2] Je.P. Volkov, E.I. Gavrilov, F.P. Duzhih, Flue pipe thermal and nuclear power stations (1987)

[3] Ya. E. Geguzin, Droplet (1973)

[4] V.I. Terehov, M.A. Pahomov, Heat and mass transfer and hydrodynamics in gas-drop flows: monograph, NGTU publishing (2008)

[5] D.V. Gvozdjakov, V.E. Gubin, On the influence of convection on the formation of drops of sulfuric acid in the atmosphere, adjacent to the area of thermal power station, Izvestia TPU, 4, 52 (2013) 
[6] D.V. Gvozdyakov, V.E. Gubin, A.A. Matveeva, On the influence of convection on the formation of droplets of sulfuric acid in the atmosphere adjacent to the location of thermal power station, EPG Web of Conference, 76 (2014)

[7] A.D. Labuncov, Physical fundamentals of Energy. Selected works on heat exchange, hydrodynamics, thermodynamics, MEI Publ. (2000)

[8] G.V. Kuznetsov, M.A. Sheremet, Mathematical modeling of complex heat transfer in a rectangular enclosure, Thermophysics and Aerodynamics, 1, 128 (2009)

[9] G.V. Kuznetsov, M.A. Sheremet, New approach to the mathematical modeling of thermal regimes for electronic equipment, Rassian Microelectronics, 2, 131 (2008)

[10] G.V. Kuznetsov, P.A. Strizhak, 3D problem of heat and mass transfer at the ignition of a combustible liquid by heater metal particle, Journal of Engineering Thermophysics, 1, 72 (2009)

[11] G.V. Kuznetsov, P.A. Strizhak, The influence of heat transfer conditions at the hot particle-liquid fuel interface on the ignition characteristics, Journal of Engineering Thermophysics, 1, 162 (2009) 